\title{
Atypical cause of hibernating myocardium due to complex cardiovascular lesions associated with Takayasu's arteritis
}

\author{
M Nakajima, K Tsuchiya, J Obata
}

Heart 2004;90:e43 (http://www.heartjnl.com/cgi/content/full/90/7/e43). doi: 10.1136/hrt.2004.036830

Myocardial hibernation is recognised as chronic hypoperfusion of the myocardium and its functional recovery after surgical revascularisation has been described. A case of surgery for complex lesions including severe aortic valve regurgitation, coronary ostial stenosis, and aortic calcification (porcelain aorta) caused by Takayasu's arteritis is presented. The onset of left ventricular functional improvement after aortic valve replacement and coronary revascularisation were indicative of preoperative atypical myocardial hibernation caused by aortic valve disease and coronary artery disease associated with Takayasu's arteritis.

A 59 year old woman was referred to our hospital for detailed examination for congestive heart failure and arteritis. She had previously noted heart murmur and diminished upper body pulse during pregnancy at the age of 23. She remained entirely asymptomatic over the next 35 years with no evidence of relapse. Ultimately, however, she presented with an approximately one year history of worsening exertional dyspnoea and chest discomfort. She was a smoker ( 10 cigarettes a day for 35 years) and severe hypertension and hyperlipidaemia were noted.

On admission, active inflammation was not noticed, with no fever and with a $\mathrm{C}$ reactive protein concentration of $2.3 \mathrm{mg} / \mathrm{l}$, but her haemodynamic condition was unstable. Chest radiography showed cardiomegaly with a cardiothoracic ratio of $59.4 \%$ and mild congestion. A transthoracic echocardiogram showed severe aortic regurgitation with left ventricular dilatation and global dysfunction (diastolic dimension of $65 \mathrm{~mm}$, systolic dimension of $51 \mathrm{~mm}$, and fractional shortening of 0.21 ). Cardiac catheterisation data showed severe systemic hypertension (238 $\mathrm{mm} \mathrm{Hg}$ ), decreased diastolic pressure $(62 \mathrm{~mm} \mathrm{Hg})$, and increased left ventricular end diastolic pressure $(47 \mathrm{~mm} \mathrm{Hg})$. Left ventriculography showed a severely dilated and dysfunctional left ventricle with an end diastolic volume index of $171.1 \mathrm{ml} / \mathrm{m}^{2}$, end systolic volume index of $119.5 \mathrm{ml} / \mathrm{m}^{2}$, and ejection fraction of $30 \%$. Neither inotropic stimulation nor stress induced evaluation of left ventricular function could be performed preoperatively because of persistently unstable haemodynamic status caused by severe aortic regurgitation. Angiographic findings were typical of Takayasu's arteritis: severe aortic regurgitation, mid left subclavian arterial stenosis, descending thoracic aortic narrowing, and stenosis of both coronary ostia. Furthermore, computed tomography showed severe aortic calcification (porcelain aorta). Early surgical intervention was indicated for these complex lesions.

At operation, the heart was approached through a median sternotomy. After institution of cardiopulmonary bypass with right atrial drainage and right axillary perfusion, the heart was arrested by antegrade infusion of blood cardioplegia. The aortic valve was replaced and double coronary artery bypass grafting (with saphenous vein grafts to the left anterior descending branch and the right coronary artery) was performed. The patient was successfully weaned from cardiopulmonary bypass with the aid of an intra-aortic balloon pump and inotropes. Weaning from ventilator support was achieved 11 hours after the operation. Intraaortic balloon pump support was required for only 17 hours. Postoperative echocardiography showed a decreased left ventricular dimension and dramatic amelioration of its functional impairment (diastolic dimension of $45 \mathrm{~mm}$, systolic dimension of $31 \mathrm{~mm}$, and fractioning shortening of 0.31 ). Postoperative aortography showed patent saphenous vein grafts to the left anterior descending branch and the right coronary artery. Left ventriculography by pulmonary arterial injection showed improved left ventricular function with an end diastolic volume index of $133.0 \mathrm{ml} / \mathrm{m}^{2}$, end systolic volume index of $40.9 \mathrm{ml} / \mathrm{m}^{2}$, and ejection fraction of $69 \%$ (fig 1). The patient was discharged from the hospital without complications and is doing well after eight months.

\section{DISCUSSION}

Myocardial hibernation is recognised as a condition of viable but underperfused myocardium, featuring left ventricular dysfunction at rest, which can be partially or completely restored after revascularisation. Reduced resting perfusion is associated with alteration of cellular contractile proteins and contributes to the pathophysiology of this process, which is potentially reversible although recovery can take months. ${ }^{1-4}$ Despite increased clinical recognition of this phenomenon, its causes-when associated with heart disease other than coronary atherosclerosis-are rarely reported. Possible causes of myocardial hibernation are arteritis and an imbalance between myocardial oxygen demand and supply due to aortic insufficiency.

Takayasu's arteritis is a chronic inflammatory disease of unknown aetiology involving the aorta and its major branches. The disease progresses at variable rates to a late sclerotic stage in which there is intimal hyperplasia, medial degeneration, and adventitial fibrosis. ${ }^{5}$ The coronary arteries are affected in less than $10 \%$ of cases; the findings and interventional strategy have been described. ${ }^{67}$ Aortic regurgitation secondary to disease of the proximal ascending aorta is seen in about one quarter of cases and the effectiveness of aortic valve replacement for such cases was reported. ${ }^{8}$ However, the surgical intervention for such complex lesions, which include severe aortic valve disease, coronary ostial stenosis, and aortic calcification (porcelain aorta), has rarely been reported.

In the present case, various cardiovascular lesions caused by Takayasu's arteritis complicated the clinical features. Although preoperative evaluation of myocardial damage was quite difficult, severe left ventricular dysfunction with dilated left ventricle caused by massive aortic regurgitation, together 

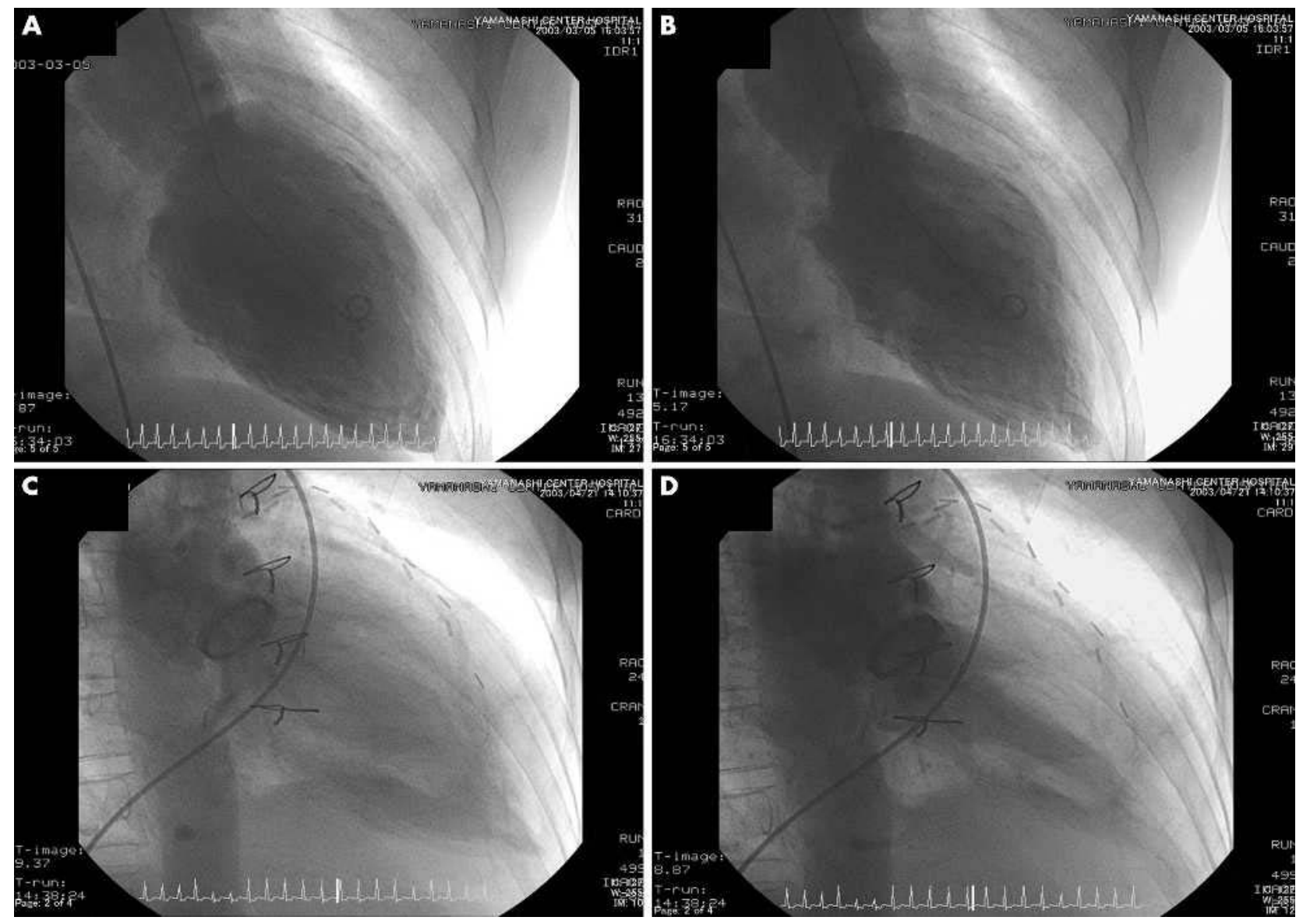

Figure 1 Left ventriculography showing improved systolic function after surgical treatment. Preoperative (A) diastolic and (B) systolic phase from right anterior oblique view. Postoperative (C) diastolic and (D) systolic phase from right anterior oblique view.

with severe stenosis of both coronary ostia, was dramatically improved by aortic valve replacement and concomitant coronary artery revascularisation. This reversible left ventricular dysfunction was considered to be atypical myocardial hibernation. That is, it was believed that prolonged hypertension and severe aortic regurgitation had gradually damaged the left ventricle, increasing its end diastolic pressure. Furthermore, combined coronary ostial stenosis compromised the blood supply to the damaged myocardium resulting in severe left ventricular systolic dysfunction with dilatation.

Surgical intervention for cases of severely dilated and dysfunctional left ventricle caused by aortic regurgitation remains controversial. Nonetheless, it may be possible to preserve myocardial function in cases complicated with coronary artery disease caused by arteritis.

\section{Authors' affiliations}

M Nakajima, K Tsuchiya, Department of Cardiovascular Surgery,

Yamanashi Central Hospital, Yamanashi, Japan

J Obata, Department of Cardiology, Yamanashi Central Hospital
Correspondence to: $\operatorname{Dr} M$ Nakajima, Department of Cardiovascular Surgery, Yamanashi Central Hospital, 1-1-1 Fujimi, Kofu city,

Yamanashi, Japan 400-0027; m-nakajima2a@ych.pref.yamanashi.jp

Accepted 17 March 2004

\section{REFERENCES}

1 Rahimtoola SH. The hibernating myocardium. Am Heart J 1989;72:211-5.

2 Elsasser A, Schleppaer M, Klovekorn WP, et al. Hibernating myocardium: an incomplete adaptation to ischemia. Circulation 1997:96:2920-31.

3 Bax JJ, Visser FC, Poldermans D, et al. Time course of functional recovery of stunned and hibernating segments after surgical revascularization. Circulation 2001; 104:1314.

4 Bax JJ, Poldermans D, Schinkel AF, et al. Perfusion and contractile reserve in chronic dysfunctional myocardium: relation to functional outcome after surgical revascularization. Circulation 2002;106:114-8.

5 Ishikawa K, Maetani S. Long-term outcome for 120 Japanese patients with Takayasu's disease: clinical and statistical analysis of related prognostic factors. Circulation 1994;90:1855-60.

6 Endo M, Tomizawa Y, Nishida $\mathrm{H}$, et al. Angiographic findings and surgical treatments of coronary artery involvement in Takayasu arteritis. J Thorac Cardiovasc Surg 2003;125:570-7.

7 Amano J, Suzuki A. Coronary artery involvement in Takayasu's arteritis: collective review and guideline for surgical treatment. J Thorac Cardiovasc Surg 1991;102:554-60.

8 Ohteki H, Itoh T, Natsuaki M, et al. Aortic valve replacement for Takayasu's arteritis. J Thorac Cardiovasc Surg 1992;104:482-6. 